Check for updates

Cite this: RSC Adv., 2019, 9, 22004

\title{
The dual deformation and remodeling of coal powders: implications for obtaining reliable stress- formed coal samples
}

\begin{abstract}
Jun Dong, ${ }^{\text {abcd }}$ Yuanping Cheng (D) *b and Pinkun Guo ${ }^{\text {abc }}$
The preparation of suitable specimens is important for obtaining credible mechanical and methane migration parameters for tectonic coal, which help to guide methane extraction and disaster prevention. In this study, a dual-deformation mechanism for porous media was introduced along with two powder compression models, and the issues that should be considered in the preparation of coal specimens were analyzed. By compression tests, the relationship between bed relative density and the applied stress in the compression of coal particles was obtained. The method of coal specimen preparation was introduced in detail. The results indicated that the Kawakita model is suitable for describing the compressive process of tectonic coal powders and guiding the preparation of tectonic coal specimens. The key parameters $a$ and $b$ in the Kawakita model are 0.411 and 0.108 , respectively. The bed relative density shows a slight increasing trend followed by an obvious rising tendency with an increase in the applied stress. A compressive stress of $150 \mathrm{MPa}$ was determined to be suitable for preparation of the tested coal specimens.
\end{abstract}

Received 22nd May 2019

Accepted 5th July 2019

DOI: $10.1039 / c 9 r a 03861 b$

rsc.li/rsc-advances commonly termed tectonic coal or tectonically deformed coal, which is widely distributed around the world and especially in China. ${ }^{11}$ Compared with tectonic coal, coal that retains its original structure or does not suffer obvious tectonism is named intact coal. Previous studies of mechanics and methane migration focused on the intact coal. It is estimated that the tectonic coal reserve is approximately 457 billion tons, accounting for $23.5 \%$ of the total coal reserves, in China. ${ }^{12}$ Other major coal-producing countries, including Britain, France, Germany, Poland, New Zealand, Russia, Turkey, Japan and Australia, have also discovered the existence of tectonic coal. Thus, it is important to pay attention to the study of tectonic coal.

Intact coal mass can be drilled, cut and polished into cylindrical samples for laboratory tests of mechanical and methane migration characteristics. Tectonic coal, however, cannot be drilled and processed into cylindrical coal samples because it is soft and easy to break. ${ }^{8}$ Therefore, the method of secondary forming is often used to prepare tectonic coal specimens. A punching machine and a mold can be used to press the tectonic coal into cylindrical samples. ${ }^{13}$ Table 1 lists the preparation information for tectonic coal samples from literature. It can be seen that researchers select different particle sizes, stress intensities, stress time and additives, and there is no universally accepted standard for tectonic coal sample preparation.

The particle size determines the raw material for preparing the coal sample, and we believe that it may be more appropriate to retain the original particle size of the tectonic coal. A longer stress time would deform the coal particles more evenly. It is acceptable to add some water as additive because the molded coal specimen 
Table 1 Preparation information of tectonic coal samples

\begin{tabular}{|c|c|c|c|c|c|c|}
\hline Sample location & $\begin{array}{l}\text { Scale } \\
(\mathrm{mm})\end{array}$ & Particle size (mm) & $\begin{array}{l}\text { Stress } \\
\text { intensity }\end{array}$ & $\begin{array}{l}\text { Stress time } \\
(\mathrm{min})\end{array}$ & Additive & Reference \\
\hline $\begin{array}{l}\text { The eighth coal mine in Pingmei } \\
\text { coal group }\end{array}$ & $\Phi 25 \times 50$ & $\begin{array}{l}\text { Combined with } 1-2 \mathrm{~mm} \text { and } 1 \\
\mathrm{~mm}\end{array}$ & $200 \mathrm{kN}$ & 20 & & 14 \\
\hline Shijiazhuang coal mine in Jincheng & $\Phi 50 \times 100$ & $0.25-0.425$ & $100 \mathrm{MPa}$ & & Water & 15 \\
\hline The eighth coal mine in Hebi & $\Phi 50 \times 100$ & $<0.5$ & $135 \mathrm{kN}$ & 30 & Water & 16 \\
\hline South of Qinshui basin & $\begin{array}{l}\Phi 24.5 \times \\
36\end{array}$ & $0.21-0.37$ & $2 \mathrm{kN}$ & 30 & $\begin{array}{l}2 \mathrm{~mL} \text { standard } \\
\text { brine }\end{array}$ & 17 \\
\hline Yushe coal mine in Guizhou & $\Phi 50 \times 100$ & $0.1-0.125,0.15-0.2,0.3-0.45$ & $100 \mathrm{MPa}$ & 20 & Water & 18 \\
\hline $\begin{array}{l}\text { No. } 1 \text { coal seam of a coal mine in } \\
\text { Guizhou }\end{array}$ & $\Phi 50 \times 100$ & $0.18-0.25$ & $200 \mathrm{kN}$ & 30 & & 19 \\
\hline Qinan coal mine in Huaibei & $\Phi 50 \times 100$ & $0.2-0.25$ & $200 \mathrm{kN}$ & 30 & Water & 20 \\
\hline Nantong coal mine & $\Phi 50 \times 100$ & $0.1-0.2$ & $100 \mathrm{MPa}$ & & & 21 \\
\hline $\mathrm{K}_{2}$ coal seam in Songzhao coal mine & $\Phi 50 \times 100$ & $0.18-0.25$ & $100 \mathrm{MPa}$ & 10 & Cement & 21 \\
\hline Zhangji coal mine in Huainan & $\Phi 50 \times 100$ & $0.18-0.425$ & $100 \mathrm{MPa}$ & & Water & 22 \\
\hline $\begin{array}{l}\text { The seventh coal seam in Datong } \\
\text { coal mine }\end{array}$ & $\Phi 50 \times 100$ & & $20 \mathrm{MPa}$ & & $2 \%$ water & 23 \\
\hline Yushe coal mine in Guizhou & $\Phi 50 \times 100$ & $0.3-0.45,0.15-0.2,0.1-0.125$ & $100 \mathrm{MPa}$ & 20 & A little water & 24 \\
\hline Zofiówka coal mine & $48 \times 48$ & $<0.2$ & $\begin{array}{l}2.76-19.9 \\
\mathrm{MPa}\end{array}$ & & A little water & 25 \\
\hline
\end{tabular}

should be dried before taking mechanical and methane migration tests. ${ }^{26}$ The compression of coal particles deforms the tectonic coal powders to form molded coal samples. Thus, the compressive stress intensity is the main parameter that influences the effects of coal sample preparation. The compressive stress intensity in Table 1 is $2.76-407 \mathrm{MPa}$, which is a wide range for us to refer to and choose a suitable value.

The reservoir conditions and mechanical properties of tectonic coal from different sources vary, so the sample preparation method should not be uniform. Different coal particle samples may have different compressive stress intensities under certain particle size and water content to obtain suitable molded coal specimens. On the other hand, the preparation of coal samples should reflect the original condition of the tectonic coal reservoir to some extent. The availability of the prepared coal specimen should be evaluated.

In this study, we analyzed the dual-deformation mechanism for porous media and the issues that should be considered in the preparation of coal specimens. Two powder compression models were introduced and compared for characterizing the compression process of tectonic coal powders. The relationship between the bed relative density and the applied stress in the process of coal particle compression was analyzed. Then, the suitable compressive stress for coal specimen preparation was determined. The obtained coal specimens were further evaluated for effectiveness using porosity tests and permeability tests.

\section{Theory}

\subsection{Powder compression models}

The tectonic coal collected from coal mines are dispersed particles. The two typical models used to characterize the compression of particulate solids are the Heckel model and Kawakita model.
In 1961, Heckel proposed that the bed relative density has the following relationship with applied stress: ${ }^{27}$

$$
\ln \frac{1}{1-\rho_{\mathrm{r}}}=A+K_{\mathrm{H}} \sigma
$$

where $\rho_{\mathrm{r}}$ is the bed relative density, which is the ratio of the final height of the agglomerate to the height during compression; $A$ is a constant; $K_{\mathrm{H}}$ is a constant, $\mathrm{MPa}^{-1}$, and its reciprocal $1 / K_{\mathrm{H}}$ is termed the Heckel parameter; $\sigma$ is the applied stress, MPa.

In the Heckel model, $\ln \left[1 /\left(1-\rho_{\mathrm{r}}\right)\right]$ and the applied stress $(\sigma)$ fit a linear relationship, and parameters $A$ and $K_{\mathrm{H}}$ can be obtained by fitting test data.

In 1970, Kawakita and Lüdde proposed the following equation: ${ }^{28}$

$$
\frac{\sigma}{\varepsilon_{\mathrm{e}}}=\frac{1}{a b}+\frac{\sigma}{a},
$$

where $\varepsilon_{\mathrm{e}}$ is the relative volume reduction, $\varepsilon_{\mathrm{e}}=\left(V_{0}-V\right) / V_{0}=$ $a b \sigma(1+b \sigma) ; V_{0}$ is the initial bed volume and $V$ is the volume at an applied stress during compression; $a$ is a constant and related to the initial bed porosity; $b$ is also a constant and its reciprocal $1 / b$ is termed the Kawakita parameter.

It can be seen that $\sigma / \varepsilon_{\mathrm{e}}$ has a linear relationship with the applied stress $(\sigma)$, and parameters $a$ and $b$ can be derived from the fitted equation. According to the initial bed height and the obtained parameters $a$ and $b$, we can further evaluate the theoretical height during compression, ultimate height, relative density and ultimate density of the bed.

\subsection{Dual-deformation mechanism for porous media}

There are two kinds of deformation mechanisms in porous media. The first is bulk deformation, which is caused by the deformation of skeleton particles (Fig. 1a). The second kind results from the relative displacement between the skeleton 

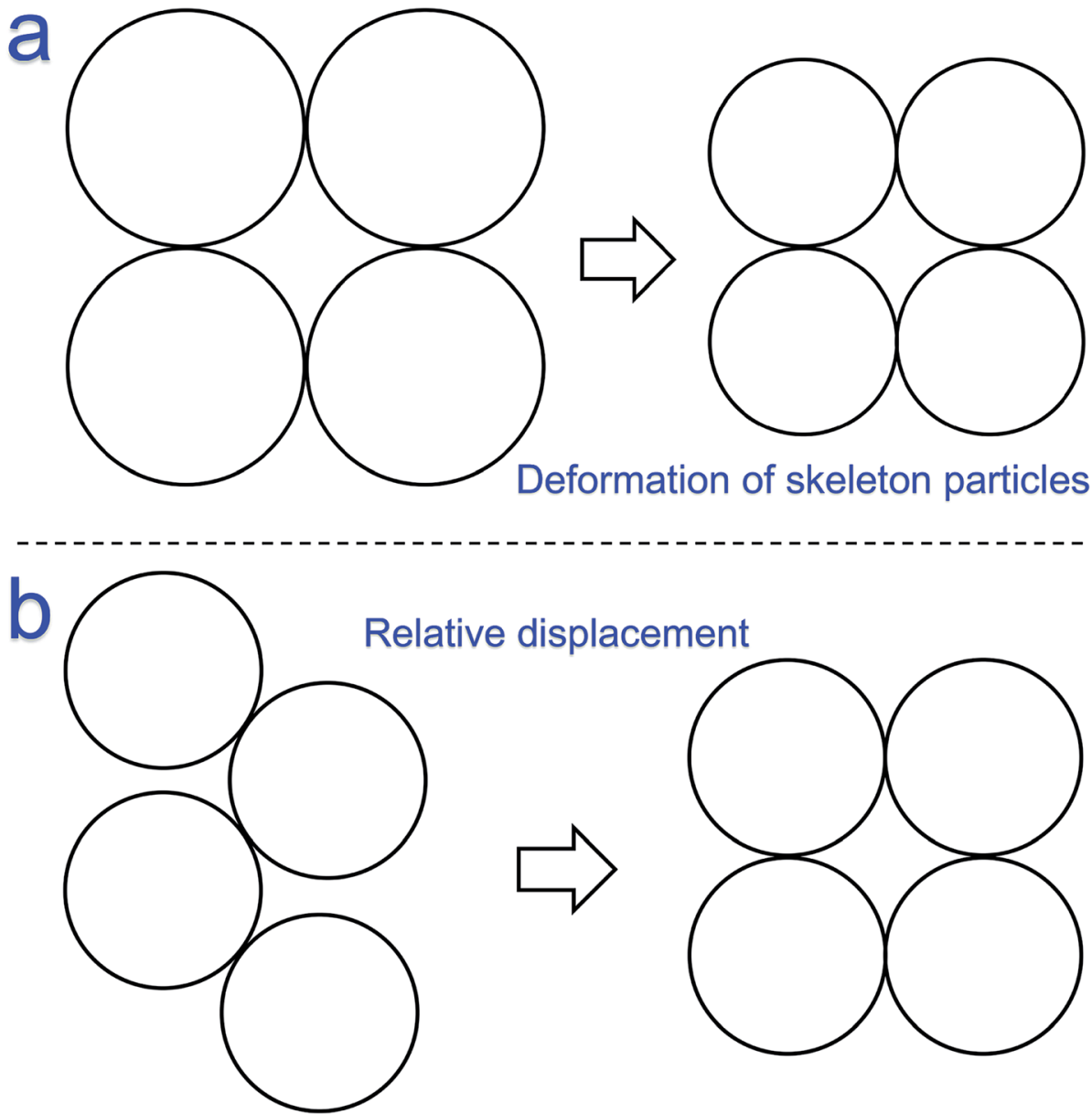

Fig. 1 A schematic diagram of the deformation of porous media: (a) bulk deformation; (b) structural deformation.

particles and is called structural deformation (Fig. 1b). ${ }^{29}$ Structural deformation is usually an irreversible, permanent plastic deformation. The fracture, viscous flow and creep of a material are the specific embodiments of structural deformation. Bulk deformation is usually a recoverable elastic deformation; the process of bulk deformation is reversible. ${ }^{30}$

The total deformation of the porous medium is the algebraic sum of the bulk deformation and structural deformation. ${ }^{31}$ The deformation response is shown in Fig. 2. When a porous medium is subjected to an external load, the bulk deformation is instantaneously completed because of the fast propagation of the stress wave that causes elastic deformation of the porous medium. However, the structural deformation persists for quite a long time after the stress is applied, because the plastic deformation response of the porous medium is determined by the unique internal structure of the medium; the structural deformation can be gradually stabilized after a long time. After applying external stress to the porous medium, the spatial structure of the particle skeleton will adjust according to the new stress conditions, and this adjustment involves the relative displacement between the particles (that is, the flow of the medium). Therefore, the structural deformation of the porous medium is a slow process. ${ }^{31}$

\section{Results and discussion}

\subsection{Considerations of coal sample preparation}

The tectonic coal collected from coal mines is fragmented and must be remodeled into cylindrical samples. The following

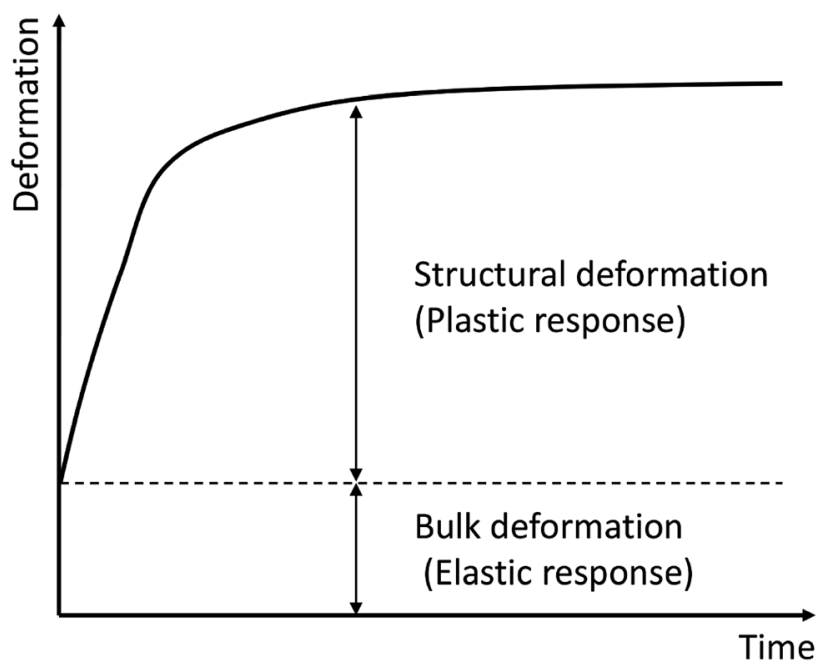

Fig. 2 A schematic diagram of the deformation response of porous medium. 
three issues need to be considered for the remodeling of tectonic coal: (1) the in situ state of the tectonic coal; (2) the state to be achieved by remodeling the tectonic coal; and (3) the compressive stress intensity for remodeling the tectonic coal.

The formation of tectonic coal involves tectonic disturbance and prolonged geostress action. The tectonic disturbance breaks the structure of coal, while the long-term geostress causes continuous relative displacement between the tectonic coal particles until the structural deformation reaches a stable state. Thus, this paper believes that the tectonic coal under in situ condition has achieved sufficient structural deformation, and the geostress can hardly cause further deformation for the tectonic coal. That is, the tectonic coal reaches a dense state.

In the process of remodeling the tectonic coal, coal powders undergo both bulk deformation and structural deformation. Samimi et al. pointed out that the deformation of particles can be separated into three stages during compression. First, the particles are subjected to sliding and rearrangement without plastic deformation at low compressive stress. Then, extensive plastic deformation and fragmentation occur in the particles with the increase in compressive stress and the decrease in bed volume. Finally, the bed shows elastic behaviour when its porosity approaches zero at high compressive stress. ${ }^{32}$ It can be concluded that the tectonic coal particles are mainly subjected to the structural deformation with relative displacement and redistribution during the compression progress. Thus, the purpose of remodeling the tectonic coal is to make the coal powders undergo a large structural deformation to achieve a basically compact and stable state.

\subsection{Model selection}

In deriving the Heckel equation, the yield stress of the material is assumed to be a constant, and the reduction in bed porosity obeys a first-order type of reaction with applied stress. ${ }^{33}$ Thus, $\ln [1 /(1-$ $\left.\rho_{\mathrm{r}}\right)$ ] has a linear relationship with the applied stress $(\sigma)$. The basis for the Kawakita equation for powder compression is that the particles subjected to compressive load are in equilibrium at all stages of compression, so that the product of the stress term and the volume term is a constant. ${ }^{34}$ Thus, $\sigma / \varepsilon_{\mathrm{e}}$ and the applied stress $(\sigma)$ fit a linear relationship. Because of the differences in model assumptions and particle properties, the linear relationship between the parameters of the two models is not always satisfied.

To evaluate whether the two models can effectively characterize the compression process of tectonic coal powders, the height of the coal bed and the simultaneous applied stress were measured with a servo testing device. Firstly, a certain mass of tectonic coal particles was weighed and placed in a mold with an inner diameter of $25 \mathrm{~mm}$. The initial height of the coal bed was measured after placing the core plug gently. Then, a constant stress increment of $10 \mathrm{~N} \mathrm{~s}^{-1}$ was loaded, and the height of the coal bed was measured intermittently. The applied stress corresponding to the height of the coal bed was also recorded until the load reached $100 \mathrm{kN}$.

Parallel experiments were carried out to obtain two sets of data for the coal bed height and applied stress. First, the Heckel model was used for the analysis, as shown in Fig. 3. It can be

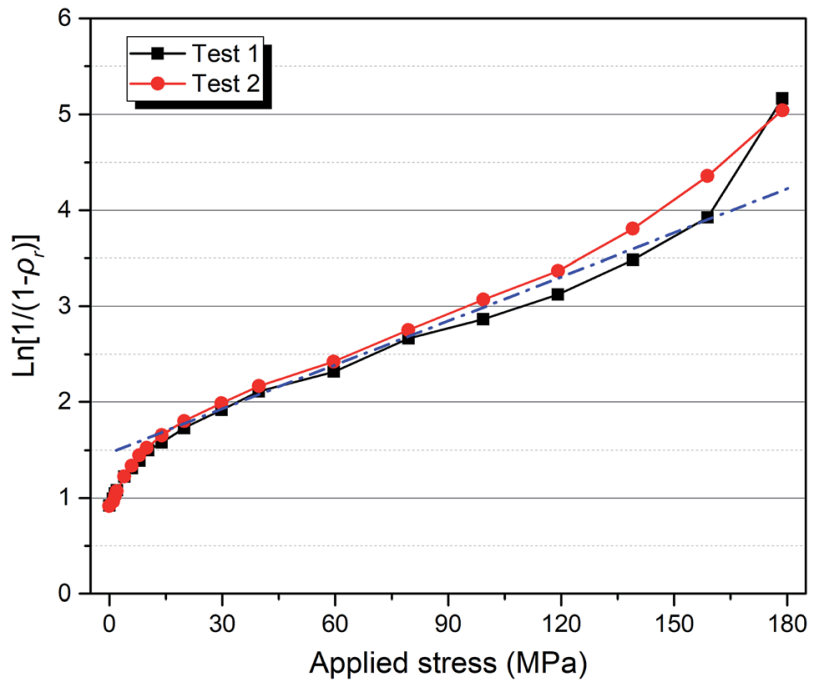

Fig. 3 Compression curves of tectonic coal using the Heckel model.

seen that a linear relationship is satisfied only in the stress range of 30-120 MPa, but there is no linear relationship for low stress or high stress. Sun et al. pointed out that the main reasons for the deficiencies in the Heckel model are: (1) sliding and rearrangement of powder particles during filling; (2) brittle fracture of powder particles at high stress; (3) the existence of particle aggregates; (4) the deficiencies of the equation itself. ${ }^{35}$ Considering the shortages of the Heckel model and the nonlinear relationship between $\ln \left[1 /\left(1-\rho_{\mathrm{r}}\right)\right]$ and the applied stress $(\sigma)$, the Heckel model was considered unsuitable to characterize the compression process of tectonic coal powders.

The $\sigma / \varepsilon_{\mathrm{e}}$ and the applied stress $(\sigma)$ in the Kawakita model was then calculated, and their relationship is illustrated in Fig. 4. The linear goodness of fit of the results for two sets of coal samples were 0.9986 and 0.9992 , and it is reasonable to conclude that the $\sigma / \varepsilon_{\mathrm{e}}$ and the applied stress $(\sigma)$ of the samples satisfies the linear relationship for the Kawakita model. Thus,

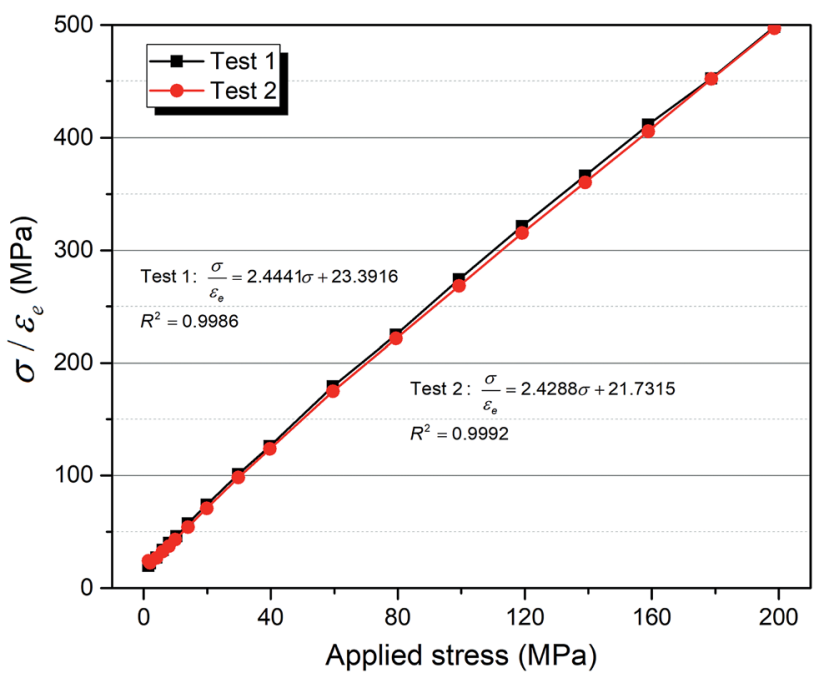

Fig. 4 Compression curves of tectonic coal using the Kawakita model. 
the Kawakita model can be used to describe the compressive process of tectonic coal powders and guide the preparation of tectonic coal specimen. It is accepted that the Kawakita model works well for soft and fluffy pharmaceutical powders. ${ }^{28,32,33}$ Thus, hard materials, such as metals, may not be suitable for this model.

\subsection{Method of coal sample preparation}

According to the fitting parameters in Fig. 4, some parameters of the Kawakita model and the coal samples can be obtained, as listed in Table 2. By substituting the average values of parameters $a$ and $b$ into eqn (2), the relationship between the bed relative density $\left(\rho_{\mathrm{r}}\right)$ and the applied stress $(\sigma)$ of the tectonic coal bed can be obtained, as illustrated in Fig. 5 .

It can be seen from Fig. 5 that the relative density of the tectonic coal bed increases rapidly at first, and then it increases slowly with the increase in the applied stress. Under the initial stress-free condition, the relative density of the tectonic coal bed is only 0.589 . When the stresses are $50 \mathrm{MPa}, 100 \mathrm{MPa}$, $150 \mathrm{MPa}$ and $200 \mathrm{MPa}$, the relative densities are $0.902,0.944$, 0.961 and 0.970 , respectively. The relative density increases rapidly when the applied stress is lower than $150 \mathrm{MPa}$. In this stage, the coal particles are loosely arranged and the void between them is large. Thus, the increasing applied stress results in extensive plastic deformation and fragmentation of the particles, and the relative density of the coal bed increases rapidly. When the applied stress is over $150 \mathrm{MPa}$, the porosity of the coal bed approaches zero, and higher applied stress can only result in tiny structural deformation. Thus, the relative density shows a slowly increasing trend. The tectonic coal bed is basically compacted to a condition close to its in situ state when the applied stress reaches $150 \mathrm{MPa}$. Thus, a compressive stress of $150 \mathrm{MPa}$ should be applied to remodel the tectonic coal specimen from the Qinan coal mine.

For other tectonic coal powders, the suitable compressive stress for remodeling the coal specimen may not be $150 \mathrm{MPa}$. Selected coal powders should be used to test and obtain the relation curve between the relative density of coal bed and the applied stress, and then the suitable compressive stress can be determined. It should be noted that the applied stress should be kept for several hours to make the coal bed cohere well and avoid damage during the demolding process.

\subsection{Effectiveness evaluation}

After obtaining the cylindrical coal specimens, we believe that some measures should be taken to evaluate their effectiveness. Because the relative density increases with the increase in

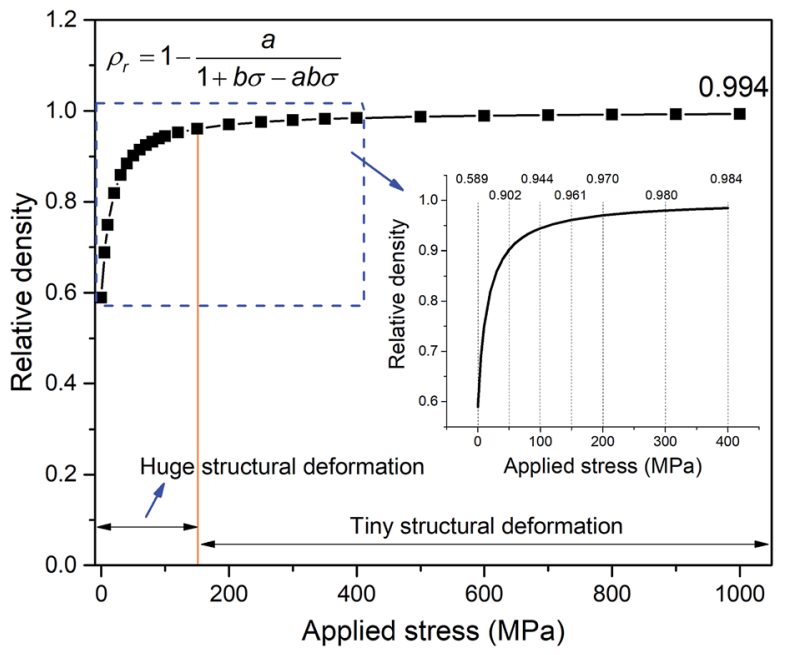

Fig. 5 Relationship between the relative density and the applied stress during the compression process of tectonic coal powders.

compressive stress, and the reduction of coal volume can be reflected in the decrease in the porosity of coal specimen, the bed porosity can be used as an evaluation parameter. A self-built coal lump porosity device (Fig. 6a) was used to test the porosities of the tectonic coal specimens.

Methane extraction from tectonic coal seams is usually found to be difficult, which may be attributed to its low permeability ${ }^{36}$ Some papers, however, found that the tested permeabilities of tectonic coal specimens are much higher than those of the intact coal specimens. The main reason may be that the remodeled coal specimens are not effective to reflect the original structure of coal. Thus, we think that the permeability can also be used to evaluate the effectiveness of the remodeled coal specimens. A coupling characteristic testing system for adsorption, seepage and mechanics of coal and rock (Fig. 6b) was used to test the permeabilities of the tectonic coal specimens.

The test results of the porosities and permeabilities under different effective stresses are listed in Table 3. For comparison, the intact coal collected near the tectonic coal was also processed into cylindrical specimens and subjected to porosity and permeability tests. The results indicated that the porosity of tectonic coal specimens is approximately 1.75 times that of the intact coal, which is basically consistent with the results of mercury intrusion, physical adsorption and methane desorption tests. ${ }^{36-38}$ The permeabilities of the prepared tectonic coal specimens are much lower than those of the intact coal specimens, which is consistent with the actual situation of methane

Table 2 Some parameters of the coal samples and Kawakita model

\begin{tabular}{|c|c|c|c|c|c|}
\hline & Weight $(\mathrm{g})$ & Diameter $(\mathrm{cm})$ & $a$ & $b$ & Limiting density $\rho_{\infty}\left(\mathrm{g} \mathrm{cm}^{-3}\right)$ \\
\hline Test 1 & 26.33 & 2.532 & 0.409 & 0.104 & 1.533 \\
\hline Test 2 & 23.32 & 2.532 & 0.412 & 0.112 & 1.534 \\
\hline
\end{tabular}




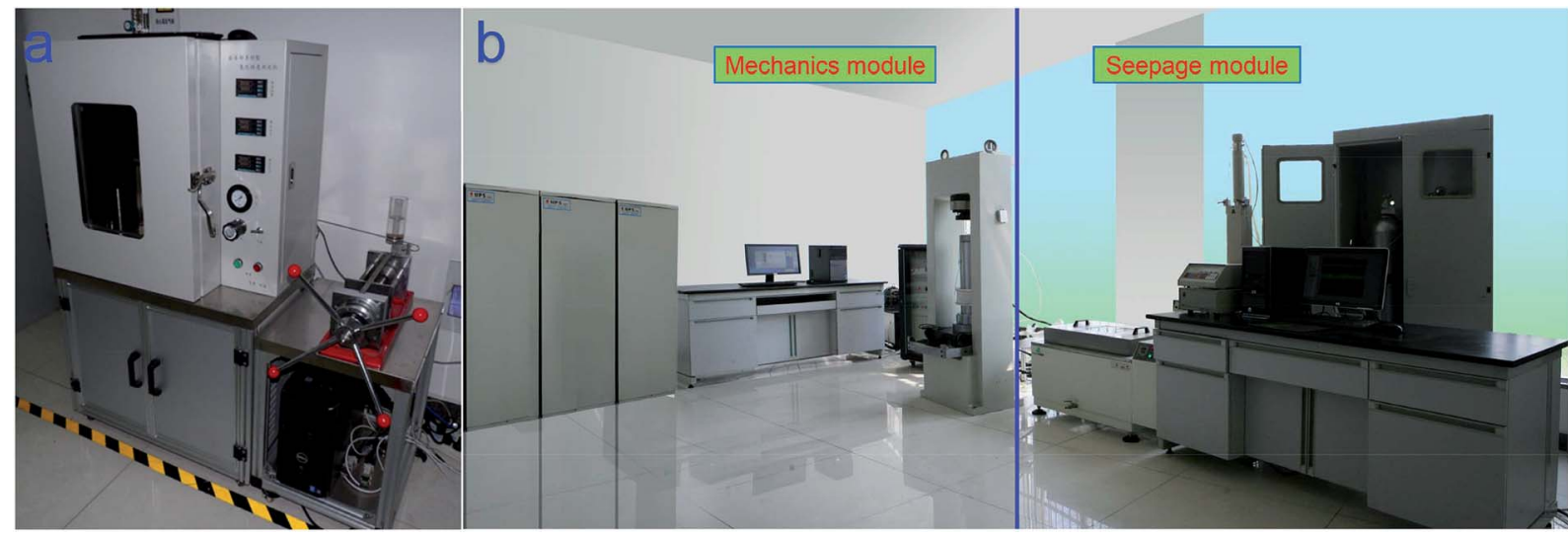

Fig. 6 Instruments for evaluating the effectiveness of coal specimens: (a) coal lump porosity device; (b) coupling characteristic testing system for examining the adsorption, seepage and mechanics of coal and rock.

Table 3 Porosities and methane permeabilities of intact coal specimens and remodeled tectonic coal specimens

\begin{tabular}{lllll}
\hline Porosity (\%) & Permeability (mD) & & \\
\hline Tectonic coal & Intact coal & Effective stress (MPa) & Tectonic coal & Intact coal \\
$11.96-12.59$ & $6.45-7.45$ & 2 & $0.0840-0.0932$ & $0.0368-5.8605$ \\
& & 4 & $0.0507-0.0509$ & $0.0122-3.5678$ \\
& & 12 & $0.0208-0.0229$ & $0.0021-0.5585$ \\
& & $0.0130-0.0136$ & $0.0010-0.1843$
\end{tabular}

extraction. ${ }^{39}$ Thus, it can be concluded that the principle and method for preparing coal specimens is effective.

\section{Conclusions}

The dual-deformation mechanism for porous media and the issues that should be considered in the preparation of coal specimens were analyzed. Two powder compression models were introduced and compared for characterizing the compression process of tectonic coal powders. A suitable compressive stress for coal remodeling was obtained by the analysis of the relationship between the relative density of the coal bed and the applied stress. A coal specimen preparation method was introduced, and the remodeled coal specimens were subjected to effectiveness evaluation using porosity tests and permeability tests. Based on this work, the following conclusions can be drawn:

(1) The coal powders undergo both bulk and structural deformation in the process of compression, and the purpose of remodeling the tectonic coal is to make the coal powders undergo a large structural deformation to achieve a basically compact and stable state.

(2) In the Kawakita equation, $\sigma / \varepsilon_{\mathrm{e}}$ and the applied stress $(\sigma)$ satisfy a linear relationship, showing that the Kawakita model is suitable for describing the compressive process of tectonic coal powders and guiding the preparation of tectonic coal specimens.

(3) With the increase in the applied stress, the bed relative density increases rapidly and then slowly. The reason for the change is that the coal powders undergo a tiny structural deformation followed by extensive plastic deformation and fragmentation.

(4) The key parameters $a$ and $b$ in the Kawakita model are 0.411 and 0.108 , respectively. A compressive stress of $150 \mathrm{MPa}$ was determined to be suitable to remodel the tectonic coal specimen from the Qinan coal mine.

(5) The suitable compressive stress for coal remodeling may not be uniform because the reservoir conditions and mechanical properties of tectonic coal vary. Selected coal powders should be used to test and obtain the relationship curve between the relative density of the coal bed and the applied stress, and then the suitable compressive stress can be determined.

\section{Conflicts of interest}

There are no conflicts to declare.

\section{Acknowledgements}

This research was supported by the Fundamental Research Funds for the Central Universities (2017CXNL02), the National Key R\&D Program of China (No. 2016YFC0800100) and the Natural Science Foundation of China (No. 51704045). 


\section{References}

1 BP, BP Statistical Review of World Energy June 2018, British Petroleum, 2018.

2 R. Kumar, R. K. Singh, A. K. Ghosh, R. Sen, S. K. Srivastava, R. S. Tiwari and O. N. Srivastava, J. Nanopart. Res., 2013, 15, 1406.

3 R. Kumar, R. K. Singh, P. K. Dubey, P. Kumar, R. S. Tiwari and I. K. Oh, J. Nanopart. Res., 2013, 15, 1847.

4 R. Kumar, R. K. Singh, P. K. Dubey, R. M. Yadav, D. P. Singh, R. S. Tiwari and O. N. Srivastava, J. Nanopart. Res., 2015, 17, 24.

5 J. Dong, Y. Cheng, K. Jin, H. Zhang, Q. Liu, J. Jiang and B. Hu, Fuel, 2017, 197, 70-81.

6 R. Pan, Y. Cheng, L. Yuan, M. Yu and J. Dong, Nat. Hazards, 2014, 73, 531-546.

7 T. Shu, D. Tang, X. Hao, L. Gao and F. Yuan, Int. J. Coal Geol., 2014, 134-135, 38-45.

8 J. Dong, Y. Cheng, B. Hu, C. Hao, Q. Tu and Z. Liu, Powder Technol., 2018, 325, 412-419.

9 J. Liu, J. Gao, X. Zhang, G. Jia and D. Wang, Rock Mech. Rock Eng., 2019, 1-19.

10 E. Wang, Q. Shao and S. Han, Coal Sci. Technol., 2009, 37, 104-106.

11 Y. Ju, B. Jiang, Q. Hou and G. Wang, J. China Coal Soc., 2004, 29, 513-517.

$12 \mathrm{H}$. Xie, Research on safety, high efficiency and green coal mining technology and strategy, Science Press, Beijing, 2014.

13 H. Chen, Y. Cheng, H. Zhou and W. Li, Rock Mech. Rock Eng., 2013, 46, 1377-1390.

14 D. Guo, C. Li and Y. Zhang, Earth Sci. J. China Univ. Geosci., 2014, 39, 1600-1606.

15 D. Zhang, Experimental study on mechanical characteristics and seepage characteristics of coal containing methane under the coupling effect between stress and thermal, Chongqing University, Chongqing, 2011.

16 C. Wang, Research on characteristics and applications of the permeability of loaded coal containing gas, Henan Polytechnic University, Jiaozuo, 2012.

17 Y. Geng, H. Xu, D. Tang, S. Tao and S. Tang, Coal Sci. Technol., 2015, 45, 197-200.
18 M. Yuan, J. Xu, B. Li, Z. Wang, Y. Du and S. Xu, Saf. Coal Mines, 2016, 47, 24-27.

19 Z. Wang and M. Yuan, China Coalbed Methane, 2017, 14, 4347.

$20 \mathrm{H}$. Chen, Damage \& permeability development of unloading coal body during mining the protective coal seam, China University of Mining and Technology, Xuzhou, 2013.

21 G. Hu, H. Wang, X. Fan and Z. Yuan, Chin. J. Rock Mech. Eng., 2009, 28, 2527-2534.

22 J. Xu, G. Ye, B. Li and J. Cao, Rock Soil Mech., 2015, 36, 104110.

$23 \mathrm{~W}$. Zhang, Study on mechanical property of soft coal under impact load and its effect to coal and gas outburst, Anhui University of Science and Technology, Huainan, 2015.

24 X. Li, G. Yin, H. Zhang and W. Wang, Chin. J. Rock Mech. Eng., 2010, 29, 3350-3358.

25 N. Skoczylas, B. Dutka and J. Sobczyk, Fuel, 2014, 134, 45-52. $26 \mathrm{X}$. Liu, Study on the deformation and failure characteristics and the permeation behavior of gas-saturated coal, China University of Mining and Technology, Xuzhou, 2013.

27 R. W. Heckel, Trans. Metall. Soc. AIME, 1961, 221, 671-675.

28 K. Kawakita and K.-H. Lüdde, Powder Technol., 1971, 4, 6168.

29 C. Li, X. Kong and X. Xu, Nat. Mag., 1999, 24, 1515-1518.

30 P. Lu, Z. Sheng, G. Zhu and E. Fang, J. Univ. Sci. Technol. China, 2001, 31, 686-693.

31 C. Li, X. Kong, Z. Du, X. Xu and P. Li, Acta Mech. Sin., 2003, 35, 230-234.

32 A. Samimi, A. Hassanpour and M. Ghadiri, Chem. Eng. Sci., 2005, 60, 3993-4004.

33 P. J. Denny, Powder Technol., 2002, 127, 162-172.

34 S. Patel, A. M. Kaushal and A. K. J. P. R. Bansal, Pharm. Res., 2007, 24, 111-124.

35 Y. Sun, H. Liu, S. Liang and P. Ding, Chin. J. Biochem. Pharm., 2013, 11, 27-34.

36 S. Lu, Y. Cheng, W. Li and L. Wang, Int. J. Oil Gas Coal Technol., 2015, 10, 91-114.

37 Z. Wang and Y. Cheng, Coal Sci. Technol., 2017, 45, 84-87.

38 Z. Wang, Y. Cheng, Y. Qi, R. Wang, L. Wang and J. Jiang, Powder Technol., 2019, 350, 15-25.

39 L. Wang and Y. Cheng, Resour. Policy, 2012, 37, 315-321. 